\title{
TU/e EmonONEN

\section{An amortized Tit-For-Tat protocol for exchanging bandwidth instead of content in P2P networks}

\section{Citation for published version (APA):}

Garbacki, P., Epema, D. H. J., \& Steen, van, M. (2007). An amortized Tit-For-Tat protocol for exchanging bandwidth instead of content in P2P networks. In Proceedings of the 1st International Conference on SelfAdaptive and Self-Organizing Systems (SASO'07, Cambridge MA, USA, July 9-11, 2007) (pp. 119-128). Institute of Electrical and Electronics Engineers. https://doi.org/10.1109/SASO.2007.9

DOI:

10.1109/SASO.2007.9

Document status and date:

Published: 01/01/2007

\section{Document Version:}

Publisher's PDF, also known as Version of Record (includes final page, issue and volume numbers)

\section{Please check the document version of this publication:}

- A submitted manuscript is the version of the article upon submission and before peer-review. There can be important differences between the submitted version and the official published version of record. People interested in the research are advised to contact the author for the final version of the publication, or visit the $\mathrm{DOI}$ to the publisher's website.

- The final author version and the galley proof are versions of the publication after peer review.

- The final published version features the final layout of the paper including the volume, issue and page numbers.

Link to publication

\section{General rights}

Copyright and moral rights for the publications made accessible in the public portal are retained by the authors and/or other copyright owners and it is a condition of accessing publications that users recognise and abide by the legal requirements associated with these rights.

- Users may download and print one copy of any publication from the public portal for the purpose of private study or research.

- You may not further distribute the material or use it for any profit-making activity or commercial gain

- You may freely distribute the URL identifying the publication in the public portal.

If the publication is distributed under the terms of Article 25fa of the Dutch Copyright Act, indicated by the "Taverne" license above, please follow below link for the End User Agreement:

www.tue.nl/taverne

Take down policy

If you believe that this document breaches copyright please contact us at:

openaccess@tue.nl

providing details and we will investigate your claim. 


\section{An Amortized Tit-For-Tat Protocol for Exchanging Bandwidth instead of Content in P2P Networks}

\author{
Paweł Garbacki and Dick H.J. Epema \\ Delft University of Technology \\ The Netherlands \\ \{p.j.garbacki,d.h.j.epema\}@tudelft.nl
}

\author{
Maarten van Steen \\ Vrije Universiteit Amsterdam \\ The Netherlands \\ steen@cs.vu.nl
}

\begin{abstract}
Incentives for resource sharing are crucial for the proper operation of $\mathrm{P} 2 \mathrm{P}$ networks. The principle of the incentive mechanisms in current content sharing $\mathrm{P} 2 \mathrm{P}$ networks such as BitTorrent is to have peers exchange content of mutual interest. As a consequence, a peer can actively participate in the system only if it shares content that is of immediate interest to other peers. In this paper we propose to lift this restriction by using bandwidth rather than content as the resource upon which incentives are based. Bandwidth, in contrast to content, is independent of peer interests and so can be exchanged between any two peers. We present the design of a protocol called amortized titfor-tat (ATFT) based on the bandwidth-exchange concept. This protocol defines mechanisms for bandwidth exchange corresponding to those in BitTorrent for content exchange, in particular for finding bandwidth borrowers that amortize the bandwidth borrowed in the past with their currently idle bandwidth. In addition to the formally proven incentives for bandwidth contributions, ATFT provides natural solutions to the problems of peer bootstrapping, seeding incentive, peer link asymmetry, and anonymity, which have previously been addressed with much more complex designs. Experiments with a real-world dataset confirm that ATFT is efficient in enforcing bandwidth contributions and results in download performance better than provided by incentive mechanisms based on content exchange.
\end{abstract}

\section{Introduction}

An important feature of peer-to-peer (P2P) architectures is that they allow to distribute the infrastructure setup and maintenance costs among the participating nodes. However, the competitive nature of $\mathrm{P} 2 \mathrm{P}$ environments encourages selfish behavior of individual peers, which has been confirmed by a number of studies. For example, it has been shown in [22] that users of a P2P system should be seen as rational agents optimizing their own performance even if their actions adversely affect the performance of other peers. In [3] it has been observed that as much as $70 \%$ of the users of a popular file-sharing P2P network are freeriders who use the system resources without giving anything in return.

The poor performance of P2P networks caused by freeriding has stimulated research on incentives for resource sharing. All incentives in one way or another translate to constraining a peer's access to the resources of others with the contributions of that peer. The fairness enforcement mechanisms proposed to date build incentives on content exchange, as contributions are always expressed in terms of the local content made available by a peer. Contentexchange incentives establish a direct (e.g., exchange of content pieces between peers in BitTorrent [7]) or indirect (e.g., exchange rings in [4] or credit paths in [15]) bartering relationship between peers that are interested in each other's content. Then, the ability of a peer to contribute resources, and consequently also a peer's access to the resources offered by others, depends on the demand on the content possessed by the peer. As a result, peers that do not offer content of immediate interest to others cannot access resources of other peers.

In this paper we propose bandwidth rather than content as the resource upon which incentives are built. Bandwidth, in contrast to content, is unrelated to the interests or tastes of a peer, and is therefore potentially a more convenient unit of trade. In the bandwidth-exchange incentive model that we propose, any peer can contribute its currently idle bandwidth to assist other peers in their ongoing downloads, regardless of the actual content that is being downloaded.

As a proof of concept, we present the design of the ATFT protocol that employs mechanisms that are conceptually similar to those in BitTorrent [7] to enforce fairness in a P2P network where peers exchange (currently) unused bandwidth rather than content. ATFT breaks the temporal nature of BitTorrent tit-for-tat (TFT) that restricts current consumptions with current contributions by allowing bandwidth consumptions to be gradually amortized over time 
with bandwidth contributions. As a side effect, in addition to fairness enforcement, ATFT offers solutions to the four fundamental problems of P2P networks of bootstrapping newcomers, of seeding incentives, of efficient use of asymmetric links, and of anonymity.

In our ATFT protocol, each peer exchanges bandwidth with a limited number of bandwidth borrowers. The members of the borrowers set are selected in a fully decentralized manner, based on the amount of bandwidth contributed in the past to the peer performing the selection. A peer uses its currently idle bandwidth to assist its borrowers in their downloads. We perform an analytical study of the ATFT protocol, formally proving its incentive mechanism and determining the impact of the protocol parameters such as the size of the borrowers set on the sharing performance. The analytical results obtained in a simplified system model are confirmed with an experimental study using an 8-week trace of a popular file-sharing P2P community, filelist.org [2].

\section{Content exchange vs bandwidth exchange}

The amount of content transferred between peers in a P2P network translates directly into the amount of bandwidth required for the transfers. However, content and bandwidth are resources with very different properties. Content has a semantic meaning attached that makes it relevant to only those peers who are interested in it. One of the consequences of the semantic context of content is that a peer is a valuable contributor only if it has content requested by the others. In a system enforcing fairness in resource sharing, not being able to contribute decreases the chances of getting access to the shared resources. This property of the content-exchange model by definition discriminates peers that do not share popular content.

The bandwidth of a peer, on the other hand, can be used by any other peer to download the content it requests. However, in contrast to content, bandwidth is not a persistent resource and therefore it cannot be exchanged as easily as content. In our previous work [10] we have introduced the concept of collaborative downloads implemented by a data transfer protocol called 2Fast that allows peers to use bandwidth of others during their downloads. In 2Fast, a downloader utilizes the idle bandwidth of helper peers to fetch the content it requests more efficiently. Helper peers do not have to be interested in, or even know what they are downloading.

Despite its limitations, content exchange rather than bandwidth exchange is the basis of all incentive mechanisms commonly used in content-sharing P2P networks. Arguably the most well-known protocol providing incentives for resource contributions in content-exchange $\mathrm{P} 2 \mathrm{P}$ networks is the BitTorrent protocol. BitTorrent uses a tit-for-tat (TFT) mechanism to prevent freeriding. BitTor- rent TFT restricts access to locally stored file pieces based on the amount of data obtained recently from the accessing peer. There are several well-known limitations of BitTorrent TFT related to the fact that its applicability is restricted to peers downloading the same content at the same time. First, the newcomers are bootstrapped at the bandwidth cost of the existing peers. Second, no incentives for content injection (seeding in BitTorrent terminology) are provided. Third, peers with asymmetric Internet connections, which constitute a majority of $\mathrm{P} 2 \mathrm{P}$ users [21], cannot fully utilize their download links as they are forced to download at the speed of their upload link.

The limitations of BitTorrent TFT are addressed to some extent in the design of credit-based systems such as $[8,11,15,23]$. However, the ability of a peer to earn credits is heavily dependent on the popularity of the content possessed by the peer. Furthermore, the sophistication of the system mechanisms required to reliably account and validate credits prevents the credit-based systems from being widely used.

In this paper we show how the limitations of BitTorrent can be overcome avoiding the complexity of creditbased systems. We propose a simple protocol using mechanisms similar to those in BitTorrent, but based on bandwidth exchange rather than content exchange.

\section{Design of ATFT}

In this section we will describe our ATFT protocol, which builds incentives for contributions on the bandwidthexchange concept. ATFT employs mechanisms similar to BitTorrent to account for a peer's bandwidth contributions and is based on those contributions to select the bandwidth borrowers with the highest chance of returning the borrowed bandwidth.

\subsection{Mechanisms of ATFT}

In this section we introduce the mechanisms of ATFT and set them off against the corresponding BitTorrent mechanisms (see Table 1). We assume here a basic knowledge of the BitTorrent mechanisms and accompanying terminology as described in, e.g., [13].

In BitTorrent, each peer maintains a limited-size set of peers interested in the file pieces possessed by the peer. The members of this set are candidates for content exchange. In ATFT, bandwidth exchange candidates are members of the borrowers set. A peer gives borrowers access to its idle bandwidth resources. The set of borrowers of a peer is not fixed, but rather it evolves based on the ability and willingness of the borrowers to return the consumed bandwidth.

A peer in BitTorrent keeps a set of peers that are potential sources of the missing pieces. The potential set is related to the interested set in the sense that peer $p$ is in the potential 


\begin{tabular}{|c|c|}
\hline ATFT & BitTorrent TFT \\
\hline borrowers set & interested set \\
\hline contributors set & potential set \\
\hline collaboration & active set \\
\hline collaborative downloads & piece exchange \\
\hline selection & choking \\
\hline exploration & optimistic unchoking \\
\hline local view & tracker \\
\hline
\end{tabular}

Table 1. The correspondence between the mechanisms of ATFT and BitTorrent TFT.

set of peer $q$ if peer $q$ is in the interested set of peer $p$. In ATFT, the equivalent of the potential set is the contributors set. Peer $p$ is a contributor of peer $q$ if peer $q$ is a borrower of peer $p$.

At any time, a BitTorrent peer downloads file pieces from the members of its potential set that have the peer in their active sets. In ATFT, a peer downloading data forms a collaboration with those among its contributors that are on-line and are not requesting themselves, and so have idle bandwidth to spare. The peer forming the collaboration is called the collector, and the contributors in the collaboration are called the helpers. The goal of the collaboration is to assist the collector in downloading the requested data using the idle bandwidth of the helpers. The collaboration is established only for the time of a single download (e.g., transfer of one file). During consecutive downloads peer roles in a collaboration may be reversed, i.e., collectors may become helpers for peers that currently act as their helpers.

Data is transferred in BitTorrent by means of piece exchange between individual peers. ATFT replaces individual peers with collaborations of collectors and their helpers. The collaborative download protocol, described in more detail in Section 3.2, defines how the idle bandwidth of the helpers is used by (or transferred to) the collector. The collaborative download protocol establishes a tit-for-tat relation between peers inside the collaboration (collector and helpers) on the one hand and peers outside the collaboration on the other. Inside a collaboration, however, data is transferred only from helpers to the collector. The view of peer roles in the ATFT protocol from the perspective of a collector is presented in Figure 1. The collaborative download protocol guarantees only that the fairness in bandwidth contributions is preserved between peers in different collaborations. It is the role of the remaining mechanisms of ATFT to ensure that also the fairness between the collector and its helpers is preserved.

Peers in BitTorrent keep track of the amount of content contributed to them by the members of the potential set. The choking and optimistic unchoking mechanisms are employed by BitTorrent to select the peers to exchange content with that result in the highest download performance.

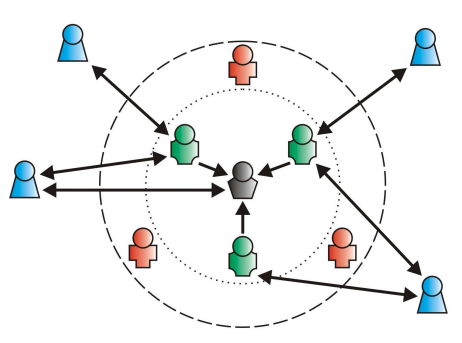

8 collector

id idle contributor acting as helper

B requesting (busy) contributor

9 non-contributor peer

$\leftrightarrow$ tit-for-tat

$\longleftarrow$ contribution from helper

collaboration

contributors set

\section{Figure 1. The collector-centric view of peer roles in the ATFT protocol.}

In ATFT, peers account and maintain the amount of bandwidth obtained from their contributions. The selection and exploration operations, described in more detail in Section 3.3, are used to select as borrowers peers with the highest contributions.

The peer discovery functionality in BitTorrent is provided by the tracker, which is a central component maintaining the IP addresses and port numbers of all connectable peers downloading a certain file. BitTorrent peers can exchange data exclusively with peers downloading the same file, and so the tracker has to keep information only about downloaders of a single file. In ATFT, bandwidth can be exchanged between any two peers, and therefore a peer should be able to discover any other peer in the network. A central peer discovery component does not scale to the entire network. Instead, we assume that a peer in the ATFT network maintains a local view of the system, which contains information about a random subset of peers. New borrowers are selected from among the peers in the local view. As long as the randomness of the peers sample in the local view is preserved, ATFT is independent of the particular protocol used to update local views. Rather than proposing a new peer sampling protocol, we point to the extensive work done in this field $[9,12]$. The size of the local view should be interpreted as the protocol configuration parameter.

\subsection{Bandwidth transfer between helpers and collector}

In BitTorrent, the (content) resource is transferred by means of transmitting pieces of a file between two peers downloading that file. In ATFT, the (bandwidth) resource transfer is handled by the collaborative download protocol that describes how the bandwidth of helpers is used by the collector. The realization of the collaborative download protocol generally depends on the type of a P2P network. Currently, the dominant $\mathrm{P} 2 \mathrm{P}$ network types are file sharing and video streaming. Collaborative downloads integrate very naturally with $\mathrm{P} 2 \mathrm{P}$ networks of both types.

In file sharing $\mathrm{P} 2 \mathrm{P}$ networks, helpers can assist the collector simply by downloading file pieces that the collector misses. To obtain new data, helpers and collector trade lo- 
cally possessed pieces for the pieces that have not yet been downloaded by any peer in the collaboration. The design of this simple protocol and its implementation in Tribler [19] $\mathrm{P} 2 \mathrm{P}$ network client have been described in detail in our previous work [10].

P2P video streaming networks require that content pieces are transferred in a certain order allowing for video playback during the download. To increase the resilience to temporal bandwidth fluctuations, video streaming protocols make use of a buffer to prefetch pieces ahead of the current playback position. Protocols as BiToS [24] employ tit-for-tat bartering for the pieces in the buffer to guarantee fairness in peer data contributions. Similarly to file sharing networks, helpers in a video streaming network could use their bandwidth to obtain missing pieces, this time restricted to the contents of the buffer.

\subsection{Forming peer relationships}

Following a rational strategy of locally optimal choices, each peer in ATFT selects as borrowers peers with the highest bandwidth contributions. The process of choosing borrowers, presented in Algorithm 1, consists of the two operations of exploration and selection, which are repeated periodically but independently of each other. The exploration and selection operations are complementary in the sense that while exploration extends the borrowers set opening opportunities for bandwidth exchange, selection reduces the borrowers set by removing the least promising peers.

The exploration performed by peer $p$ (line 1 ) is intended to expand its borrowers set up to the predefined size limit (line 2). New borrowers are selected randomly from the local view of peer $p$ (line 3). If $p$ selects peer $q$ then $q$ is added to the borrowers set of $p$ (line 4 ) and $p$ is added to the contributors set of $q$ (line 5). Execution of line 5 requires network communication between $p$ and $q$.

The selection operation (line 6) executed by peer $p$ starts with replacing the borrowers set with peers that contributed a positive amount of bandwidth to $p$ (line 7). By doing so, the peer follows the principle of reciprocation, giving access to the local bandwidth to those who contribute their own bandwidth. At this point the size of the borrowers set may be larger than allowed. In that case, we reduce the size of the borrowers set in two steps. First, we rank borrowers based on their bandwidth contributions (line 8). Second, we remove from the borrowers set all except at most $n_{p}-r_{p}$ peers with the highest contributions (lines 9 and 10).

Algorithm 1 provides high-level pseudocode that can be further customized by selecting values of the parameters $n_{x}$ and $r_{x}$ as well as the contributors ranking method. The selection of $n_{x}$ is discussed in more detail in Section 4.3. The parameter $r_{x}$ controls the "aggressiveness" of the exploration process. Generally, by setting $r_{x}$ to a higher value, more peers can be probed for their suitability as desired bor-

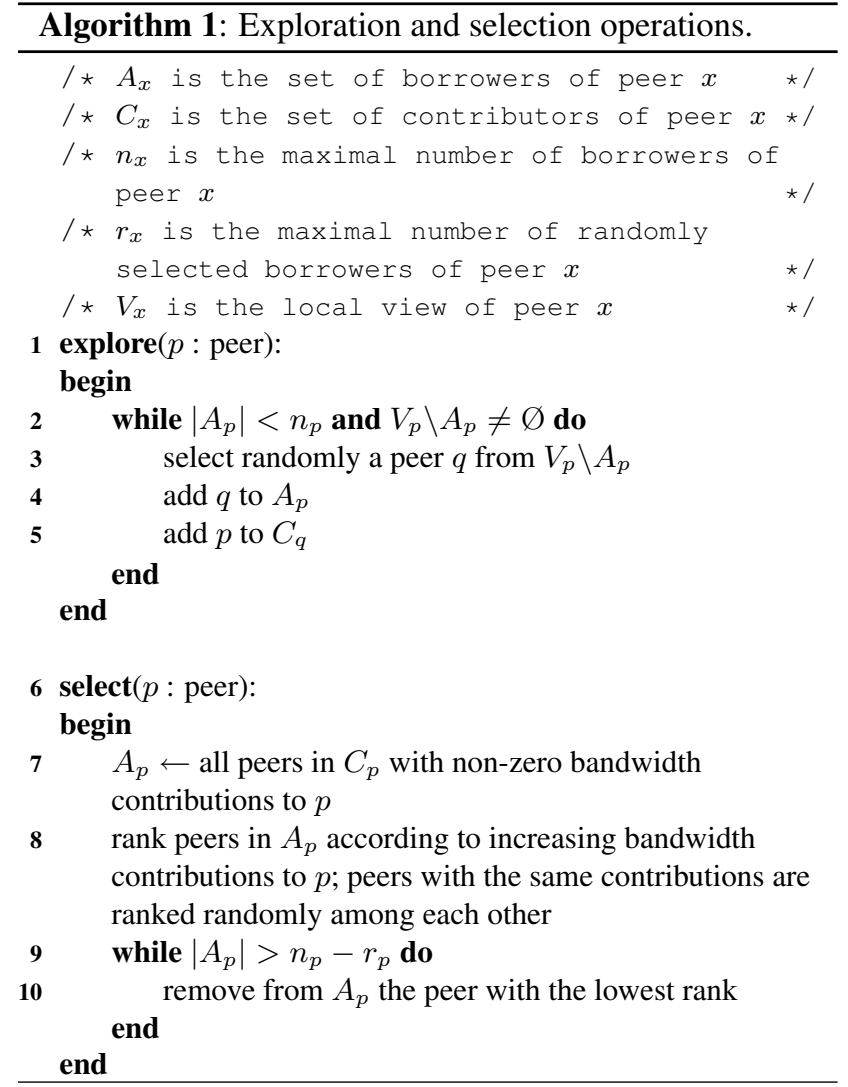

rowers in a shorter time, and consequently an optimal set of borrowers maximizing peer's gain can be found faster. On the other hand, however, increasing $r_{x}$ gives more peers access to local bandwidth resources increasing the chance that the consumed bandwidth will not be returned. Therefore, the value of $r_{x}$ should be customized depending on a peer's objective.

The formula used to compute peer contributions in line 8 of Algorithm 1 defines the borrowers selection criterion. A simple formula could just compute the total amount of bandwidth contributed by a peer, giving more recent contributions a higher weight. More complex formulas could also interpret the reliability of the contributor expressed by the probability that the contributor will act as a helper for a longer period of time. Contributor reliability aspects are extremely important in case of video streaming protocols where not only the amount of available bandwidth but also the continuity of the contribution impacts the service quality.

\subsection{Benefits of ATFT}

After presenting the design of ATFT, we will discuss how the bandwidth exchange model of ATFT addresses the four limitations of the content exchange model of BitTorrent TFT briefly mentioned in Sections 1 and 2 . 
The primary motivation behind the BitTorrent mechanism design is to prevent freeriding. Nonetheless, it has been shown that it is still possible to download data from the BitTorrent network at a reasonable speed without uploading any data in return [14]. As explained in [18], a fundamental flaw of BitTorrent's incentive mechanism is the presence of significant altruism in the protocol operation. A newcomer peer obtains the first few pieces from peers entrusting the newcomer to return the consumed bandwidth. The cost of bootstrapping is borne by the community, not the newcomer, as rationality would suggest. As part of the optimistic unchoking algorithm, peers donate usually $20 \%$ of their upload link capacity to bootstrap newcomers and find new peers to barter with. Although ATFT allows for bandwidth donation to randomly selected borrowers, the maximal number of random borrowers ( $r_{x}$ in Algorithm 1 ) is only a tuning parameter which can be set to 0 without affecting the principles of system operation. In case of $r_{x}$ equal to 0 , newcomers have to first prove themselves by contributing bandwidth to their borrowers before they will be offered access to bandwidth resources of the others. Hence, the cost of bootstrapping in ATFT is paid by the newcomers themselves, not the community.

Second, another well-known shortcoming of BitTorrent systems is the lack of incentives for seeding, i.e., for uploading content after completing a file download. Seeding is another, apart from bootstrapping of newcomers, altruistic type of behavior, which is crucial for BitTorrent system healthiness. In ATFT, seeding may be seen as a form of helping in download. The bandwidth used for seeding content to a borrower can be reclaimed in the future. Furthermore, if a helper already possesses the file requested by the collector, it does not have to reserve part of its upload capacity for tit-for-tat bartering in order to obtain file pieces from peers outside the collaboration. A seeding helper can transfer data to the collector at a speed of its upload capacity limit and be acknowledged for higher contribution than helpers that have to divide their upload link capacity between tit-for-tat and sending data back to the collector. In principle, a seeding helper contributes to the collector twice as much data as a helper using tit-for-tat to obtain missing file pieces. In conclusion, ATFT provides a clear incentive for content seeding.

Third, as explained in [10], BitTorrent cannot utilize the download link capacity of upload-capacity-limited peers. The temporal nature of BitTorrent TFT allows a peer to download only with the speed of its upload link capacity. ATFT detaches in time the periods when the bandwidth is contributed from the periods when the bandwidth is consumed. Consequently, peers can fill their down links completely with the bandwidth of the helpers.

Finally, the bandwidth-exchange model of ATFT can be used to provide a certain level of anonymity. A collector can decide not to expose its identity outside a collaboration by downloading data exclusively from the helpers. Since the identity of the collector is still known to members of the collaboration, helpers can be then seen as anonymizing proxies [1].

\section{Analysis of ATFT}

In this section we analyze the mechanisms of ATFT that provide incentives for bandwidth contributions and we investigate the impact of the size of the borrowers set on the bandwidth sharing performance. We start with a description of the P2P network model used in our analysis.

\subsection{System model}

In our model of a P2P system for analyzing ATFT, we denote by $N$ the total number of peers in the system. All peers maintain a borrowers set of the same size $n$. We perform our analysis under steady state, assuming that all the peers have already found stable sets of borrowers. We do not study the impact of the number of borrowers that are selected randomly $\left(r_{x}\right.$ in Section 3.3) on the performance of ATFT.

We assume that peers are alternating between idle periods and request periods, and that the durations of both types of periods are exponentially distributed, with the same means for all peers. Let the average length of the idle periods and requesting periods be $1 / \lambda$ and $1 / \mu$, respectively. In this analysis we assume that the idle bandwidth of every helper is distributed evenly among its requesting borrowers.

The contribution of peer $i$, denoted by $c_{i}$, is equal to the average amount of bandwidth given to the borrowers during its idle periods. The contribution is bounded by a value dependent on the upload link capacity of the peer. The gain $g_{i}$ of peer $i$ is the average amount of bandwidth obtained by peer $i$ from its contributors.

Furthermore, we assume that each peer can use as much help as it can get. Although the link capacity limit bounds the number of helpers that suffice to fill a peer's download link, using more helper peers improves the resilience to content availability changes caused by, e.g., transmission failures or peer leaves. This type of resilience is crucial in video streaming P2P networks.

\subsection{Incentive for contribution}

We prove analytically that in ATFT, a peer's gain grows when its own contribution grows. ATFT thus provides a direct incentive for bandwidth contributions. In our analysis, we exploit the fact that the mechanisms of ATFT closely resemble those of BitTorrent. Therefore we can directly apply the analytical methodology developed for BitTorrentlike systems to show certain properties of ATFT.

An analytical model of BitTorrent-like systems has been introduced in [20]. This model is based on assumptions 
analogous to those described by us in Section 4.1. Namely, the model in [20] assumes that peers request files to download according to a Poisson process and that they stay in the system for a random time which is exponentially distributed. BitTorrent peers have global knowledge about other peers in the system. The capacity of a BitTorrent peer is expressed by its upload link limit. No download bandwidth constraints are imposed. The contribution in BitTorrent is defined as the amount of data transferred to a peer in the active set in a recent time interval. The gain of a BitTorrent peer is the amount of data obtained from other peers in the same time interval.

ATFT extends the contribution accounting model of BitTorrent by allowing contributions to be maintained across multiple downloads. When peer contributions are computed, the mechanisms performing the selection of the best peers to barter with in BitTorrent and the selection of borrowers in ATFT operate on the same principles. Hence, also the incentive mechanisms built into the peer selection methods in BitTorrent and ATFT correspond to each other.

Before formulating the properties of the ATFT incentive mechanism, we introduce some conventions and notations in addition to those described in Section 4.1. Let's assume that peers are arranged in decreasing order according to their bandwidth contributions. Each peer is assigned a number in $1,2, \ldots, N$ representing its position in this ordering. Let $g(c)$ denote the average gain of peers with contribution $c$. The following corollary describes the correlation between the contribution and the gain of a peer in ATFT.

Corollary 1. Suppose that peers $i, i+1, \ldots, j$ have the same contribution $c$, where $i$ (respectively $j$ ) is the first (respectively last) peer with this contribution. Then for any $k>j$, we have $g_{i} \geq g_{i+1} \geq \cdots \geq g_{j} \geq g_{k}$. If additionally $j+i-1>n \geq 2$, then $g(c)>g_{k}$.

Proof. Analogous to the proof of Lemma 2 in [20].

According to Corollary 1, the ATFT protocol guarantees that if the contribution of peer $p$ is greater than the contribution of peer $q$, then the gain of peer $p$ is at least as large as the gain of peer $q$. Furthermore, with the exception of a few special cases, the gain of peer $p$ is greater than the gain of peer $q$. Hence, the ATFT protocol awards peers for their contributions by assigning better borrowers to the peers that contribute more.

We have shown that peers with higher contributions gain at least as much as peers with lower contributions. However, at this point it is not clear if a peer can preserve its current gain while decreasing its amount of contributed bandwidth. Now we will investigate how the bandwidth sharing strategy, i.e., the rules for choosing the amount of bandwidth contributed to borrowers, affects a peer's gain.

In [20], the problem of selecting the optimal bandwidth sharing strategy is modeled as a competitive game. A peer's

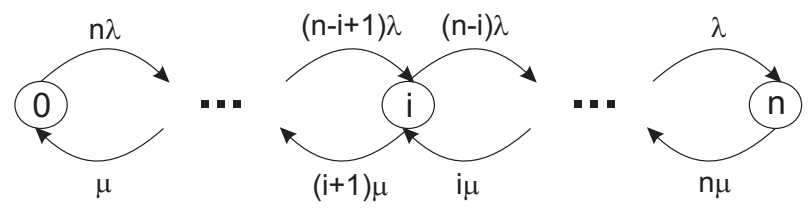

Figure 2. Evolution of the number of request-
ing borrowers.

goal in this game is to maximize its gain (primary objective) while minimizing its contribution (secondary objective). The set of optimal bandwidth sharing strategies for all peers joinly is given by a Nash equilibrium [16]. It is shown in [20] that if upload link capacities of peers are different, a Nash equilibrium may not exist. Therefore, let's consider a network with a finite number of peer bandwidth groups with all peers in a bandwidth group having the same upload bandwidth limit. Note that this is in fact a realistic assumption in the current Internet that consists of a limited number of link capacity classes. The following corollary characterizes the existence and the form of a Nash equilibrium.

Corollary 2. If $n \geq 2$ and if the number of peers in each bandwidth group is greater than $n+1$, there exists one and only one Nash equilibrium in which the contribution of each peer is equal to its upload bandwidth limit.

Proof. A direct consequence of Proposition 1 in [20].

Corollary 2 states that the only optimal strategy for a peer maximizing its gain is to contribute the entire available upload bandwidth.

\subsection{Borrowers set size}

The size of the borrowers set is an important parameter of the ATFT protocol. We analyze the impact of the borrowers set size on the sharing performance.

As we have formally proven in Section 4.2, an optimal bandwidth sharing strategy for a peer is to contribute to its borrowers as much bandwidth as possible. So it is in the best interest of a peer to maximize the utilization of its idle bandwidth. The utilization of bandwidth is defined as the fraction of the idle time when the bandwidth is (completely) used by at least one borrower. It is obvious that the utilization depends on the size of the borrowers set. We denote by $u_{n}$ the utilization when the borrowers set has size $n$.

Bandwidth utilization depends on the probability that at least one borrower requests bandwidth at a given time. The evolution of the number of requesting borrowers can be modeled as a birth-death process represented by a Markov chain, as depicted in Figure 2. The state of this process represents the number of requesting borrowers. 
If $\pi_{i}$ is the probability of state $i$, then the balance equations of this Markov chain are

$$
(n-i) \lambda \pi_{i}=i \mu \pi_{i+1}, \quad i=0, \ldots, n-1,
$$

from which we find:

$$
\pi_{i}=\left(\begin{array}{c}
n \\
i
\end{array}\right)\left(\frac{\lambda}{\mu}\right)^{i} \pi_{0} .
$$

The probability $\pi_{0}$ is determined by the normalization condition $\sum_{i=0}^{n} \pi_{i}=1$ :

$$
\pi_{0}=\left(1+\frac{\lambda}{\mu}\right)^{-n}
$$

Since we assume that the bandwidth is fully utilized as long as there is at least one requesting borrower, the bandwidth utilization can be expressed as:

$$
u_{n}=1-\pi_{0} .
$$

Substituting for $\pi_{0}$ the value computed in Eq. 1 we get

$$
u_{n}=1-\left(1+\frac{\lambda}{\mu}\right)^{-n} \text {. }
$$

According to Eq. (2), the bandwidth utilization converges very fast and is high even for small values of $n$. Keeping the borrowers set small has a practical reason discussed in [5]. Every new peer accepted as a borrower increases the chance that the contributed bandwidth will not be returned. A borrower refusing to return the contributed bandwidth will be eventually removed from the borrowers set, but the bandwidth it consumed cannot be recovered. It is therefore important for each peer to restrict the borrowers set to a number of valuable peers just large enough to fill the peer's download capacity.

\section{Performance evaluation}

In this section we present an experimental evaluation of the ATFT protocol. We first describe the experimental setup and then demonstrate the results of the experiments.

\subsection{Experimental setup}

We model the P2P network used in our experiments using a trace of a popular file-sharing P2P community, filelist.org [2]. The properties of the trace are presented in Table 2. Two reasons motivated the selection of the filelist.org trace as input for our experiments. First, filelist.org employs BitTorrent as the data distribution protocol. Because BitTorrent is currently the dominant $\mathrm{P} 2 \mathrm{P}$ protocol [17], we believe that filelist.org users are representative of average P2P network users. Second, filelist.org provides detailed user information allowing us to track users

\begin{tabular}{|c|c|}
\hline Property & Value \\
\hline trace start date & December 10, 2005 \\
\hline trace end date & January 31, 2006 \\
\hline number of peers (users) & 91,169 \\
\hline number of files & 4,021 \\
\hline average file size & $1,084 \mathrm{MB}$ \\
\hline number of file downloads & $2,326,187$ \\
\hline
\end{tabular}

Table 2. Filelist.org dataset properties.

\begin{tabular}{|c|c|c|}
\hline \multicolumn{2}{|c|}{ Bandwidth capacity } & \multirow{2}{*}{ Fraction of peers } \\
\hline Download & Upload & \\
\hline $784 \mathrm{kbps}$ & $128 \mathrm{kbps}$ & 0.2 \\
\hline $1500 \mathrm{kbps}$ & $384 \mathrm{kbps}$ & 0.4 \\
\hline $3 \mathrm{mbps}$ & $1 \mathrm{mbps}$ & 0.25 \\
\hline $10 \mathrm{mbps}$ & $5 \mathrm{mbps}$ & 0.15 \\
\hline
\end{tabular}

\section{Table 3. Bandwidth distribution of peers used in our experiments.}

across downloads of different files, a task that is generally hard for BitTorrent communities that do not provide such detailed user information.

The filelist.org dataset contains the meta-information describing the files requested by the peers, such as file size, and times of the requests. However, the dataset does not include the specification of peer bandwidth settings. We use the peer bandwidth distribution model introduced in [6]. According to this model, peers are divided into four bandwidth classes with probabilities as presented in Table 3 .

We have developed a discrete time simulator of the ATFT protocol. In the simulations, we replay the system usage scenario of each peer, assuming that a peer is requesting bandwidth for a time period required to download the entire file, and is idle otherwise. The time when the file is requested and the size of the file are given in the filelist.org dataset. The download time depends on the download bandwidth of the peer during the requesting period. The download bandwidth of a collector is the sum of the bandwidths provided by the helpers and the bandwidth the collector obtains by bartering its upload bandwidth with peers outside the collaboration. Helpers divide their bandwidth resources evenly among the requesting borrowers. This means in particular that a peer can act as a helper for multiple borrowers at the same time, with each of those borrowers getting the same amount of the peer's bandwidth. In our simulations we assume a strict tit-for-tat so there are no altruistic peers such as seeds willing to donate their bandwidth.

In the beginning of the simulation, the borrowers set of each peer is filled with randomly selected peers. As motivated in Section 4, ATFT peers do not have to maintain large borrowers sets. In our simulation we set the borrowers set size to 30 . The number of borrowers selected 


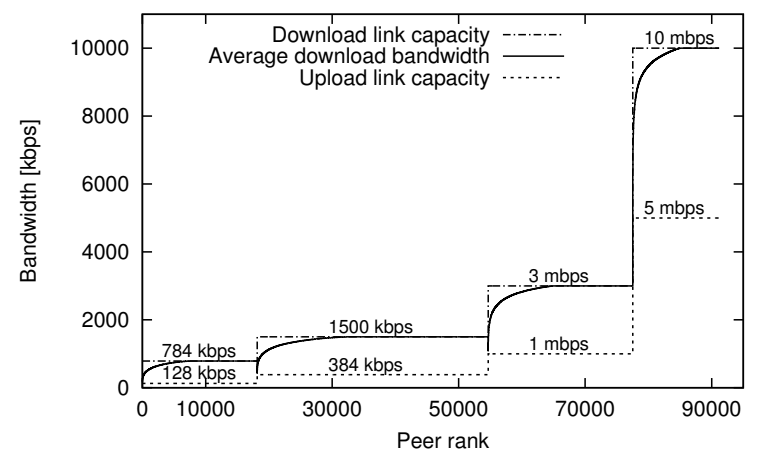

Figure 3. The average download bandwidth during request periods.

randomly equals 3 . The exploration and selection operations are performed every 10 minutes. To eliminate the bias caused by the random initialization of the borrowers sets, we start collecting data for a peer after its borrowers set has been updated 20 times.

As explained in Section 3.3, there are different ways of accounting for peer bandwidth contributions. In our simulations, a peer maintains a single value for keeping track of the bandwidth obtained from each contributor. This value is increased for every unit of bandwidth (e.g., one byte) received from the corresponding contributor, and it is cut in half every time the selection algorithm is executed to give a higher weight to more recent contributions.

\subsection{Results of the experiments}

We have conducted several experiments to assess different performance aspects of the ATFT protocol and compare it with the performance of BitTorrent-like systems. The results of our experiments allow us to quantify the performance improvement achieved by replacing the contentoriented incentive model of BitTorrent with the bandwidthoriented model of ATFT.

\subsubsection{Download performance}

In the first series of experiments we investigate the download performance improvement by using the additional bandwidth offered by the contributors. All peers apply the optimal bandwidth sharing strategy as described in Section 4.2, giving their borrowers access to the entire upload bandwidth available during idle periods.

Figure 3 presents the average download bandwidth for each peer measured during its request periods. Here, peers are grouped according to their upload and download link capacities. The groups represent the link capacity classes described in Table 3. Obviously, the download bandwidth of a peer is always between its upload and download link capacity. Peers in each link capacity class are ranked according to their average download bandwidth. The results

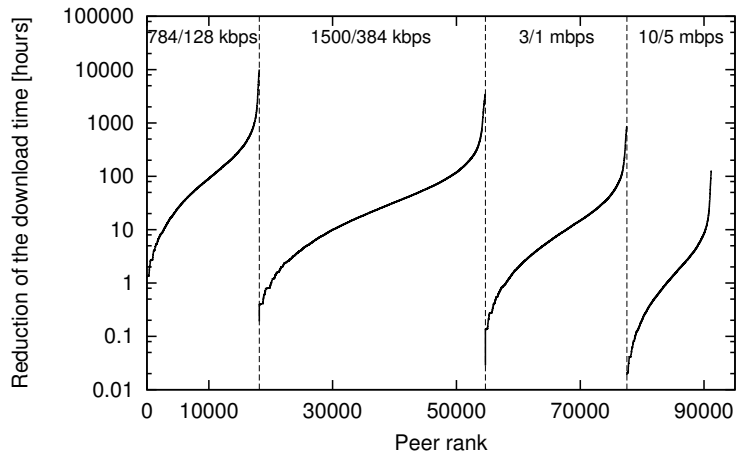

\section{Figure 4. The reduction of the total down- load time when using ATFT instead of BitTor- rent TFT.}

in Figure 3 indicate that ATFT leads to a high utilization of peer download links. On average, the download links of more than $90 \%$ of the peers are completely filled during more than $85 \%$ of the request periods. Note that in a system in which peers exchange content using BitTorrent TFT, a peer's download bandwidth is limited by its upload link capacity. We conclude that ATFT improves the average download bandwidth of a peer by a factor of 2 to 6 , depending on the link capacity asymmetry.

The increase of the download bandwidth does not say much about the benefit in terms of the reduction in time spent downloading, which depends also on the amounts of data requested by peers. Therefore, in Figure 4 we present for each peer the difference between the time spent downloading requested files using the ATFT protocol and the time required to download the same files using BitTorrent TFT over the length of the entire trace. Also in this figure, the peers are grouped based on their bandwidth classes and ranked in each group according to the download time reduction. It can be observed that for some peers the reduction in download time is greater than the duration of the entire trace. To explain this anomaly we point out that the bandwidth limit assigned to a peer by the simulator may not match the actual bandwidth of the peer, which is impossible to extract from the filelist.org dataset. Nevertheless, the results presented in Figure 4 are still indicative if we assume that peers request files independently of their bandwidth limits, and that they are determined to wait until the requested files have been downloaded.

\subsubsection{Contributing borrowers}

In ATFT, peers contribute bandwidth to their borrowers with the intention to reclaim this bandwidth in the future. The exploration and selection algorithms of ATFT follow a simple strategy of giving access to local bandwidth to peers that contributed most in the past. In the ideal case, a peer should contribute bandwidth only to those peers that recip- 


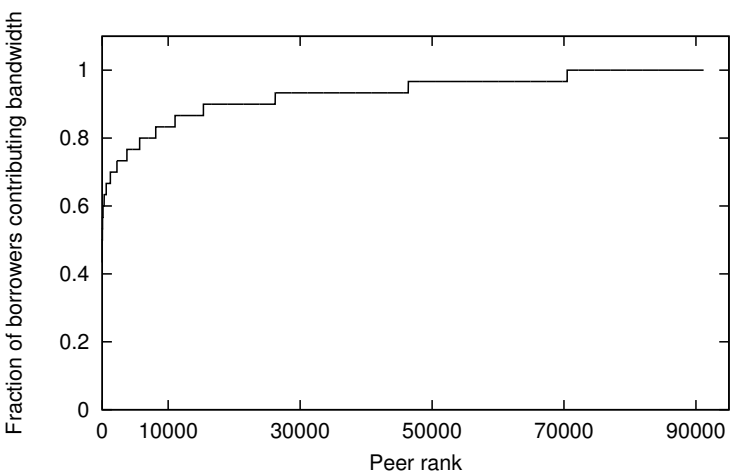

Figure 5. The fraction of borrowers contributing bandwidth averaged over peer requesting periods.

rocate by giving the peer access to their own bandwidth resources. In other words, ideally the borrowers and the contributors sets of a peer should be the same. We evaluate experimentally how effective the exploration and selection mechanisms described in Section 3.3 are in finding borrowers that return the contributed bandwidth.

Figure 5 shows the average fraction of borrowers that are also contributors. The average has been computed by summing the fractions of contributing borrowers measured for all requesting periods and dividing the sum by the number of those periods. For $90 \%$ of peers, more than 25 out of the total of 30 (a fraction of 0.83 ) borrowers are contributing bandwidth. Furthermore, on average the number of contributing borrowers of a peer is equal to 27 which is also the number of non-randomly selected peers in Algorithm 1.

\subsubsection{Freeriding}

The main purpose of incentives in $\mathrm{P} 2 \mathrm{P}$ networks is to stimulate contributions. In the following experiment we investigate the possibility of freeriding in ATFT. Freeriders consume the bandwidth resources of their contributors but refuse to help the others by keeping their borrowers sets empty. The bandwidth gain of a freerider obviously depends on the number of borrowers selected randomly by non-freeriding peers (see also Sections 3.3 and 3.4). We compare the bandwidth gain of freeriders and non-freeriders assuming that the role of freerider is assigned to $50 \%$ of randomly and uniformly selected peers.

Figure 6 presents the average bandwidth gain, i.e., the bandwidth contributed by helpers during requesting periods of freeriders and non-freeriders as it depends on the number of borrowers selected randomly $\left(r_{x}\right)$. There are a few observations that can be made here. First, although setting $r_{x}$ to 0 effectively reduces the gain of freeriders, it also negatively affects the gain of non-freeriders. In general, the process of finding optimal borrowers may be slow if $r_{x}$ equals 0 . Nonetheless, ATFT offers a reasonable performance to

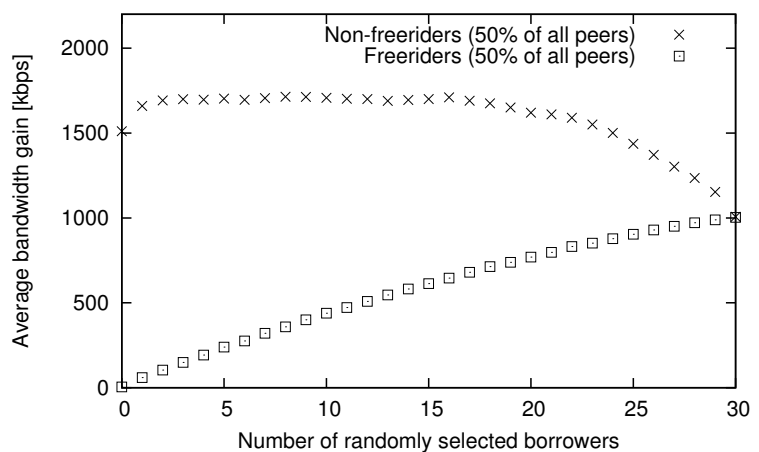

Figure 6. The average bandwidth gains of freeriders and non-freeriders as functions of the number of borrowers selected randomly.

non-freeriders even when $r_{x}$ equals 0 . In contrast, BitTorrent TFT requires that the number of randomly selected peers to upload data to is always positive (see Section 3.4).

Second, the bandwidth gain of non-freeriding peers starts decaying only if $r_{x}$ exceeds a certain threshold, in our experiment equal to 16 . Until this threshold is reached, the gain of non-freeriders stays roughly at the same level. The system capacity expressed in terms of the bandwidth available during the idle periods of non-freeriders is sufficient to tolerate freeriding to some extent. The excess system capacity allows peers to set $r_{x}$ higher, potentially increasing the chance of finding better borrowers faster, without decreasing their bandwidth gain, even in the presence of freeriding.

\subsubsection{Sensitivity to the size of the borrowers set}

In the final experiment we investigate the impact of the borrowers set size on the bandwidth gain. Based on the results of the analysis performed for a simplified system model we concluded in Section 4.3 that peer's gain is close to optimal already for small sizes of the borrowers sets. Here we validate the correctness of this conclusion in a system model derived from a real-world trace.

Figure 7 presents the correlation between the average bandwidth gain, computed in the same way as in Section 5.2.3, and the size of the borrowers set. Here the number of randomly selected borrowers is set to the smallest integer value larger than or equal to one tenth of the borrowers set size. The experimental evaluation confirms the results obtained analytically that the gain as a function of the borrowers set size converges quickly.

\section{Conclusions and future work}

In this paper we have proposed to replace the traditional incentive model of P2P networks which is based on content exchange with a novel incentive model based on bandwidth exchange. Bandwidth as a resource has a universal value for all the peers, unlike content, which is relevant only to 


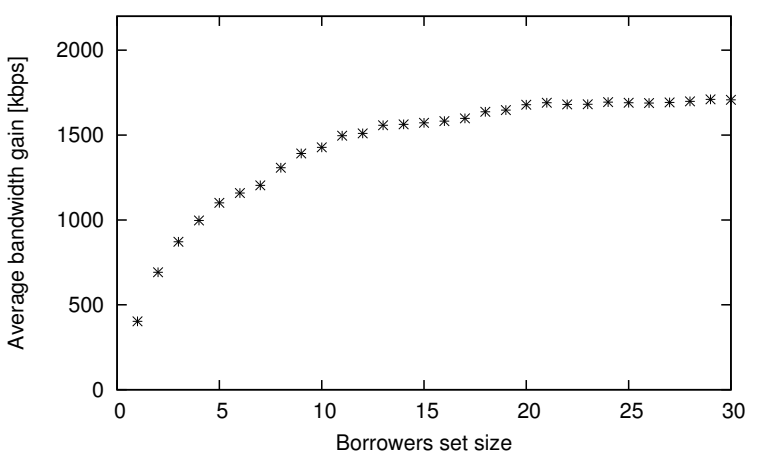

Figure 7. The average bandwidth gain as a function of the size of the borrowers set.

the group of peers interested in it. Therefore, the mechanisms for keeping track of the bandwidth exchanged between peers can be simpler than the mechanisms for maintaining the content contributions of peers across multiple downloads. As a proof of the bandwidth exchange concept, we have designed an ATFT protocol based on mechanisms employed by BitTorrent TFT for content exchange. ATFT offers an elegant solution to the problems encountered in BitTorrent including bootstrapping of newcomers, providing seeding incentive, efficient support of asymmetric links, and anonymity. We have formally proven that the ATFT protocol provides incentives for contributing bandwidth, discussed the selection of protocol parameters, and evaluated ATFT using a trace of a real-world P2P community.

We are currently working on the implementation of the ATFT protocol as part of the Tribler [19] system. This implementation will allow us to investigate the effect of the bandwidth-exchange incentive model on the behavior of users. In particular, it will be interesting to see if users leave their P2P software connected to the network in idle mode for periods longer than currently, to help their borrowers and consequently also to improve the performance of their own downloads.

\section{References}

[1] Anonymizer project page. http://anonymizer.com/.

[2] Filelist project page. http://filelist.org/.

[3] E. Adar and B. A. Huberman. Free riding on gnutella. Technical report, Xerox PARC, August 2000.

[4] K. G. Anagnostakis and M. Greenwald. Exchange-based incentive mechanisms for peer-to-peer file sharing. In ICDCS'04, Tokyo, Japan, March 2004.

[5] R. Axelrod. The Evolution of Cooperation. Basic Books, August 1985.

[6] A. R. Bharambe, C. Herley, and V. N. Padmanabhan. Analyzing and improving a bittorrent network's performance mechanisms. In Infocom 2006, Barcelona, Spain, April 2006.
[7] B. Cohen. Incentives build robustness in bittorrent. In P2PEcon'03, Berkeley, CA, May 2003.

[8] Y. Fu, J. Chase, B. Chun, S. Schwab, and A. Vahdat. Sharp: an architecture for secure resource peering. In SOSP'03, New York, NY, 2003.

[9] A. J. Ganesh, A. M. Kermarrec, and L. Massoulie. Peer-topeer membership management for gossip-based protocols. IEEE Transactions on Computers, 52(2), 2003.

[10] P. Garbacki, A. Iosup, D. Epema, and M. van Steen. 2fast: Collaborative downloads in $\mathrm{p} 2 \mathrm{p}$ networks. In 6th IEEE Int'l Conference on Peer-to-Peer Computing (P2P2006), Cambridge, UK, September 2006.

[11] M. Ham and G. Agha. Ara: A robust audit to prevent freeriding in $\mathrm{p} 2 \mathrm{p}$ networks. In 5th IEEE Int'l Conference on Peer-to-Peer Computing (P2P2005), 2005.

[12] M. Jelasity, R. Guerraoui, and A.-M. Kermarrec. The peer sampling service: Experimental evaluation of unstructured gossip-based implementations. In Middleware'04, Toronto, Ontario, Canada, October 2004.

[13] A. Legout, G. Urvoy-Keller, and P. Michiardi. Rarest first and choke algorithms are enough. In IMC'2006, Rio de Janeiro, Brazil, October 2006.

[14] N. Liogkas, R. Nelson, E. Kohler, and L. Zhang. Exploiting bittorrent for fun (but not profit). In IPTPS'06, Santa Barbara, CA, February 2006.

[15] A. Nandi, T.-W. Ngan, A. Singh, P. Druschel, and D. S. Wallach. Scrivener: Providing incentives in cooperative content distribution systems. In Middleware'05, Grenoble, France, November 2005.

[16] J. F. Nash. Equilibrium points in n-person games. Natl Acad Sci U S A, 36(1), January 1950.

[17] A. Parker. The true picture of peer-to-peer file-sharing, panel presentation. In IEEE 10th Int'l Workshop on Web Content Caching and Distribution (WCW 2005), Sophia Antipolis, France, September 2005.

[18] M. Piatek, T. Isdal, T. Anderson, A. Krishnamurthy, and A. Venkataramani. Do incentives build robustness in bittorrent? In NSDI'07, Cambridge, MA, April 2007.

[19] J. Pouwelse, P. Garbacki, J. Wang, A. Bakker, J. Yang, A. Iosup, D. H. J. Epema, M. Reinders, M. van Steen, and H. Sips. Tribler: A social-based peer-to-peer system. Concurrency and Computation: Practice and Experience, accepted 2007.

[20] D. Qiu and R. Srikant. Modeling and performance analysis of bittorrent-like peer-to-peer networks. SIGCOMM Comput. Commun. Rev., 34(4), October 2004.

[21] S. Saroiu, K. P. Gummadi, and S. D. Gribble. A measurement study of peer-to-peer file sharing systems. In MMCN'02, San Jose, CA, January 2002.

[22] J. Shneidman and D. Parkes. Rationality and self-interest in peer to peer networks. In IPTPS'03, Berkeley, CA, February 2003.

[23] V. Vishnumurthy, S. Chandrakumar, and E. G. Sirer. Karma: A secure economic framework for $\mathrm{p} 2 \mathrm{p}$ resource sharing. In P2PEcon'03, Berkeley, CA, June 2003.

[24] A. Vlavianos, M. Iliofotou, and M. Faloutsos. Bitos; enhancing bittorrent for supporting streaming applications. In 9th IEEE Global Internet Symposium (GI2006), Barcelona, Spain, April 2006. 